Eli Lilly, Janssen Pharmaceuticals, MSD, Novartis, Pfizer, Roche, Sanofi, and UCB Pharma., Speakers bureau: Pfizer, AbbVie, Amgen, UCB, BMS, Biogen, MSD, Novartis, Eli Lilly and Company, and Janssen Pharmaceuticals, Anders Odgaard: None declared, Søren Overgaard: None declared, Lene Dreyer Consultant for: MSD, UCB and Janssen Pharmaceuticals, Speakers bureau: MSD, UCB and Janssen Pharmaceuticals, Speakers bureau: UCB, MSD, Eli Lilly and Janssen Pharmaceuticals.

DOI: 10.1136/annrheumdis-2019-eular.490

\section{OP0092 ASSOCIATIONS BETWEEN CLINICAL VARIABLES AND PSYCHOLOGICAL SYMPTOMS IN RHEUMATOID ARTHRITIS: A NETWORK SCIENCE PERSPECTIVE}

Hsiu Yen Tung ${ }^{1}$, Sam Norton ${ }^{1}$, Faith Matcham ${ }^{1}$, James Galloway ${ }^{1}$, Matthew Hotopf ${ }^{1} .{ }^{1}$ King's College London, London, United Kingdom

Background: Rheumatoid arthritis (RA) is associated with an increased prevalence of common mental disorders, including anxiety and depression (Matcham et al. 2013). Borsboom's (2017) network theory of mental illness is gaining traction as a model for depression which incorporates biological, social and psychological aspects of depression by looking at interactions between individual symptoms and other variables within a wider network. Currently applications have been restricted to psychiatric samples, however, this model may potentially help gain a clearer understanding of the causal links clinical variables and mental health symptoms in RA.

Objectives: To study the prescriptions of NSAIDs in patients with rheumatoid arthritis (RA), psoriatic arthritis (PsA) and ankylosing spondylitis (AS) registered in ICEBIO and matched controls, and explore their relationship with disease activity measures. In addition, to explore the impact of initial TNF $\alpha$-inhibitor therapy on NSAID prescription rates.

Objectives: To test the feasibility of a network analysis approach to examine associations between clinical variables and mental health symptoms in RA.

Methods: The data used are from patients attending rheumatology clinics at King's College Hospital who completed patient reported outcomes (PROs) electronically via the Integrating Mental and Physical Healthcare (IMPARTS) system. Over 1,000 patients completed PROs via IMPARTS, with a subsample of 211 extracted for this analysis where psychological screening and inflammatory markers were recorded concurrently (<14days). The screening tools used were the two-item Patient Health Questionnaire (PHQ2) and the two-item Generalised Anxiety Disorder (GAD2), which assess the core symptoms of depression and anxiety: low pleasure/interest, low mood, high anxiety, uncontrollability of worry. Additional data recorded were joint counts and visual analogue scales for pain, fatigue and global disease activity. Missing data were imputed using multiple imputation. Network analysis was conducted using the ggraph package in $\mathrm{R}$ based on the regularised correlations between variables. With a graphical network model of variables created to calculate centrality values.

Results: Figure 1 below illustrates that the symptoms with the most connections were PHQ1 (low pleasure/interest), GAD2 (uncontrollable worry), pain and global disease activity. As expected the strongest connections were between PHQ1 (low pleasure/interest) with PHQ2 (low mood), GAD1 (high anxiety) with GAD2 (uncontrollable worry), pain with patient global, tender joints with swollen joints, and ESR with CRP.

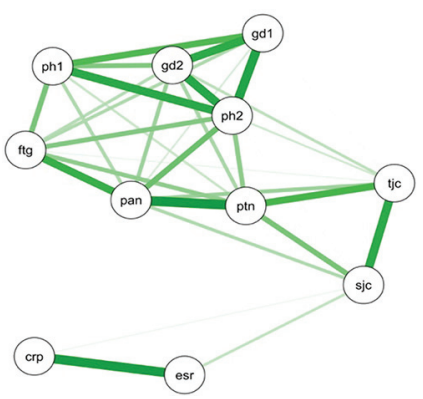

The results highlight pain and PHQ2 (low mood) as having both the highest degree (3.9 \& 3.8, respectively) and betweenness centrality ( $22 \& 10$, respectively). This indicates that these are the variables with both the highest number of connections and providing the shortest pathway between other symptoms and so may act as key variables linking inflammation and mental health. Pain and global disease activity had the highest closeness centrality $(0.033 \& 0.032$, respectively), illustrating that they have the shortest path with all other symptoms and capture the influence of both inflammation and mental health. Tender and swollen joints have weak connections to the mental health variables, suggesting that that extraarticular aspects of pain may be important.
Conclusion: Inflammation in RA does not appear to have a strong influence on mental health, with pain providing the main connection between these areas of the network. Concerning the symptoms of mental health considered, all were strongly connected but low mood provided the main connection between clinica and psychological variables. This indicates mood as potentially a key variable in $\mathrm{RA}$, which is easy to monitor in routine care.

Disclosure of Interests: Hsiu Yen Tung: None declared, Sam Norton: None declared, Faith Matcham: None declared, James Galloway Consultant for: Pfizer Inc, Matthew Hotopf: None declared

\section{REFERENCES:}

Borsboom, D. (2017). A network theory of mental disorders. World Psychiatry 16(1), 5-13. doi:10.1002/wps.20375Matcham, F., Rayner, L., Steer, S., \& Hotopf, M. (2013). The prevalence of depression in rheumatoid arthritis: a sys tematic review and meta-analysis. Rheumatology (Oxford), 52(12), 2136-2148. doi:10.1093/rheumatology/ket169 DOI: 10.1136/annrheumdis-2019-eular.2095

\section{OP0093 THE INCIDENCE AND RISK FACTOR OF NEW CAROTID PLAQUES AND THE PROGRESSION RATE OF CAROTID PLAQUES IN PATIENTS WITH RHEUMATOID ARTHRITIS IN 6 YEARS PROSPECTIVE CASE CONTROL STUDY. -TOMORROW STUDY-}

Shohei Anno $^{1,2}$, Kentaro Inui $^{2}$, Tadashi Okano ${ }^{2}$, Kenji Mamoto $^{2}$, Yuko Sugioka ${ }^{3}$, Masahiro Tada ${ }^{4}$, Tatsuya Koike ${ }^{3,5}$, Hiroaki Nakamura ${ }^{2}$. ${ }^{1}$ Osaka social medical center, Orthopaedic Surgery, Osaka, Japan; ${ }^{2}$ Osaka City University Graduate School of Medicine, Orthopaedic Surgery, Osaka, Japan; ${ }^{3}$ Osaka City University Graduate School of Medicine, Center for Senile Degenerative Disorders (CSDD), Osaka, Japan; ${ }^{4}$ Osaka City General Hospital, Orthopaedic Surgery, Osaka, Japan; ${ }^{5}$ Search Institute for Bone and Arthritis Disease (SINBAD), Shirahama, Japan

Background: Cardiovascular disease is one of the complications of rheumatoid arthritis (RA). Patients with RA show higher rates of cardiovascular disease mortality and overall mortality compared with individuals without RA. The presence of an abnormally increased intima-media thickness (IMT) in the carotid artery and carotid plaques on carotid ultrasound are useful for assessing the presence of subclinical atherosclerosis. A greater presence of carotid artery IMT and carotid plaques is a predictor of cardiovascular disease events.

Objectives: The objective of this study was to evaluate progression of carotid plaques in 6 years by comparing 2011 and 2017, and to assess the risk factors of progression.

Methods: This study included 208 patients with RA and 204 age- and sexmatched controls (Co) in the TOtal Management Of Risk factors in Rheumatoid arthritis patients to IOWer morbidity and mortality (TOMORROW) study. This was a 10-year cohort study that started in 2010. Carotid ultrasound was performed in 2011 and 2017. Ultrasound examination of bilateral carotid arteries was performed using high-resolution B-mode ultrasound (HI VISION Avius; Hitachi Aloka Medical, Tokyo, Japan) with a 6- to $18-\mathrm{MHz}$ liner array transducer. IMT was evaluated as the distance between the luminal-intimal interface and the medialadventitial interface. IMT was measured using two calipers on the frozen frame of a suitable longitudinal image. The upper limit of normal for IMT was defined as 1.0 $\mathrm{mm}$. Lesions with any focal structure that protruded into the vessel lumen for at least an IMT $>1.1 \mathrm{~mm}$ were defined as atherosclerotic plaques. Subsequently, the plaque score was assessed as the sum of the maximal thicknesses of all plaques in bilateral carotid arteries in the scanning area. Plaque scores were categorized as follows: none, no plaques; mild, score of 1.1-5.0; moderate, score of 5.110.0; and severe, score of $>10.0$.

Results: A total of 175 patients with RA (mean age: $58.9 \pm 12.7$ years, female ratio: $85.7 \%$, mean disease duration: $15.0 \pm 11.7$ years) and $185 \mathrm{Co}$ (mean age: $58.5 \pm 12.5$ years, female ratio: $84.3 \%$ ) were finally analyzed. Carotid plaques were observed more frequently in the RA group than in the Co group in 2011 ( $n=$ 82 vs $n=66, p=0.04)$. However, the incidence of new plaques was not significantly different between the RA and Co groups $(n=33$ vs $n=44, p=0.94)$. Age ( $p$ $=0.015)$ and the presence of diabetes $(p=0.009)$ were higher in patients with RA and new plaques than in those without new plaques. Multivariate logistic regression analysis did not show that RA was a risk factor for the incidence of new plaques (OR: $0.90,95 \% \mathrm{Cl}: 0.47-1.73, \mathrm{p}=0.750$ ). However, the presence of hypertension (OR: $3.11,95 \% \mathrm{Cl}: 1.43-6.74, \mathrm{p}=0.004)$, the presence of diabetes (OR: $6.13,95 \% \mathrm{Cl}: 1.13-33.4, \mathrm{p}=0.036$ ) were risk factors for the incidence of new plaques. The plaque score became advanced in the RA and Co groups (both $\mathrm{p}<0.001$ ) in 6 years.

Conclusion: There are no significant differences in the incidence of new plaques and the progression rate of the plaque score between patients with RA and Co. This finding might due to the recent advances in RA treatment, such as methotrexate or biologic DMARDs. In patients with RA, the presence of hypertension and diabetes represents a risk factor for the incidence of new plaques. 
Acknowledgement: We wish to thank Atsuko Kamiyama, Tomoko Nakatsuka and the members of the Osaka City University hospital clinical research center. Disclosure of Interests: Shohei Anno: None declared, Kentaro Inui Speakers bureau: Takeda Pharmaceutical, Pfizer Japan, Daiichi-Sankyo Co.Ltd., Abbvie, Mitsubishi Tanabe Pharma Corporation, Janssen Pharmaceutical, Chugai Pharmaceutical, Ono Pharmaceutical, Eisai Co.Ltd., Eli-Lilly, Nippon Kayaku Co.,Ltd., Maruho Co.,Ltd, Kaken Pharmaceutical Co.,Ltd., Tadashi Okano Speakers bureau: AbbVie, Kenji Mamoto: None declared, Yuko Sugioka: None declared, Masahiro Tada Speakers bureau: Abbvie, Astellas Pharma, Bristol-Myers Squibb, Chugai Pharmaceutical, Eisai, Janssen Pharmaceutical, Mitsubishi Tanabe Pharma Corporation, Ono Pharmaceutical, Pfizer Japan, Takeda Pharmaceutical, Tatsuya Koike Speakers bureau: AbbVie, Astellas Pharma Inc., Bristol-Myers Squibb, Chugai Pharmaceutical, Eisai, Janssen, Lilly, Mitsubishi Tanabe Pharma Corporation, MSD, Ono Pharmaceutical, Pfizer, Roche, Takeda Pharmaceutical, Teijin Pharma, and UCB, Hiroaki Nakamura: None declared

DOI: 10.1136/annrheumdis-2019-eular.2925

\section{OP0094 SECULAR TRENDS IN THE INCIDENT RISK OF CEREBROVASCULAR ACCIDENT IN RHEUMATOID ARTHRITIS RELATIVE TO THE GENERAL POPULATION}

Kiana Yazdani ${ }^{1}$, Hui Xie ${ }^{1}$, Antonio Aviña ${ }^{1}$, Yufei Zheng ${ }^{1}$, Michal Abrahamowicz ${ }^{2}$, Diane Lacaille ${ }^{1} .{ }^{1}$ Arthritis Research Canada, Richmond, Canada; ${ }^{2}$ Mc Gill University, Montréal, Canada

Background: Recent studies have demonstrated a declining trend in RA mortality relative to the general population (1). This improvement in mortality could be due to improvement in incident risk of cardiovascular events that are the leading cause of excess deaths in RA (2).

Objectives: Our objective was to assess secular trends in ten-year incident risk of cerebrovascular accident (CVA) in incident cohorts of RA versus general population controls, using administrative health data

Methods: We conducted a retrospective study of a population-based cohort of incident RA cases who first met previously published RA criteria between 01/01/ 1997 and 31/12/2004 in British Columbia followed until 31/12/2014, with general population controls matched 2:1 on gender, age, and index year. Individuals were excluded if they had a diagnosis of CVA prior to index date. Incident CVA was defined as first CVA during follow-up using ICD codes 9 code 433, 434/ICD-10 code 164, 163) in Hospital Discharge data or death certificate in Vital Statistics data. RA and general population cohorts were stratified according to year of RA incidence, defined according to first RA visit, using a 7-year wash-out period. Incident rates (IRs) of CVA for RA and general population cohorts, as well as incident rate ratios (IRRs), with $95 \%$ confidence intervals $(\mathrm{Cl})$ were calculated per calendar years of incidence. Multivariable Cox Proportional Hazard models with left truncation were used to estimate risk of CVA in RA relative to general population while controlling for potential confounders, with contribution of person time of follow-up starting from index date (second RA visit) to avoid immortal time bias and censoring at ten years from incident year, or last health care utilization. To examine whether secular trends differed in RA relative to general population, an interaction term was tested between the RA indicator and year of RA incidence. To account for non-linear effect of cohort year, we compared cox regression models with linear, quadratic, and flexible spline forms of the cohort-year effects and the model with the best AIC was used to interpret the data.

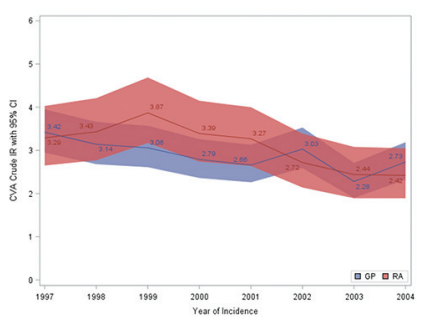

Results: 23,545 RA individuals (65.7\% female; mean [SD] age 58.11[16.82] years) and 47,090 controls experienced 658 and 1,220 incident CVA respectively. A linear spline Cox model with a knot at year 1999 was selected to fit the CVA events. The change in CVA risk over time differed significantly in RA vs. general population after 1999 [ $p=0.0488]$, but not before 1999 [ $p=0.06]$. A significant decline in risk of CVA was observed over the calendar years of incidence after 1999 in RA [0.91 $(0.86,0.96) ; p=.0003]$ but not in the general population [0.97 $(0.93,1.01) ; p=0.1019]$.

Conclusion: Our findings suggest that the risk of CVA has significantly declined over time in people with RA onset from 1999 onwards, but not in the general population.

\section{REFERENCES:}

[1] Lacaille, D., et al., Improvement in 5-year mortality in incident rheumatoid arthritis compared with the general population - closing the mortality gap. Annals of the Rheumatic Diseases, 2016.

[2] Myasoedova, E. and S.E. Gabriel, Overview of rheumatoid arthritis and mortality in relation to cardiovascular disease, in Handbook of Cardiovascular Disease Management in Rheumatoid Arthritis, A.G. Semb, Editor. 2017, Springer International Publishing: Cham. p. 1-17.

Disclosure of Interests: Kiana Yazdani: None declared, Hui Xie: None declared, Antonio Aviña: None declared, Yufei Zheng: None declared, Micha Abrahamowicz: None declared, Diane Lacaille Grant/research support from: Bristol-Myers Squibb and Eli Lilly Canada

DOI: 10.1136/annrheumdis-2019-eular.6398

\section{OP0095 INFLUENCE OF PERIODONTITIS ON DISEASE ACTIVITY, PHYSICAL FUNCTION, AND SAFETY IN PATIENTS WITH RHEUMATOID ARTHRITIS: A OBSERVATIONAL STUDY USING THE IORRA COHORT}

Mayuko Hayashi, Ryoko Sakai, Eiichi Tanaka, Takefumi Furuya, Eisuke Inoue, Mai Abe, Mika Kawano, Eri Sugano, Naohiro Sugitani, Kumiko Saka, Moeko Ochiai, Yoko Shimizu, Rei Yamaguchi, Naoki Sugimoto, Katsunori Ikari, Atsuo Taniguchi, Masayoshi Harigai, Hisashi Yamanaka. Tokyo Women's Medical University, Department of Rheumatology, Tokyo, Japan

Background: Periodontitis (PD) is considered to be one of the triggers for rheumatoid arthritis (RA) ${ }^{1}$. Several reports demonstrated the associations between the disease activity of RA and presence of PD, however, most of them are based on small population, and results are inconsistent ${ }^{2,3}$. Furthermore, impact of PD on physical function and safety is not known. Thus, a study using a large cohort database is warranted to clarify the relationship between patients' outcomes and PD among patients with RA.

Objectives: To demonstrate the influence of PD on the outcome of RA, an established cohort IORRA database was used to compare the disease activity, physical function and prevalence of infection between patients with PD and those without.

Methods: IORRA database is an established cohort database with RA in our institute since 2000. Trough biannual data collection including patient's questionnaire, physician's evaluations and laboratory data in more than 5,000 RA patients a database with a total 91,884 patient-year observation period was established by 2018. In this IORRA database, RA patients who answered to all the questionnaires about PD in October 2016 were extracted. Among those, we defined patients with PD (PD group) as having diagnosis of PD during the last 6 months, and those without PD (non-PD group) as having no present and previous PD. Using the data set from April 2016 to October 2016, we compared Disease Activity Score 28 (DAS28), Japanese Health Assessment Questionnaire (J-HAQ) score and the prevalence of patients self- reported infections required hospitalizations or hospital visits between the two groups. For background data comparisons, we used chi-squared test for categorical data and Mann-Whitney U-test for continuous data. To investigate associations between PD and remission or PD and infection, we calculated adjusted odds ratio (OR) of PD using a logistic regression model.

Results: At baseline, patients in the PD group $(n=925)$ were significantly older, had higher DAS28 and J-HAQ than those in the non-PD group $(n=2,583)$. DAS28 and J-HAQ at 6 month in the PD group were significantly higher than those of the non-PD group (DAS28, 2.60 in PD group, 2.42 in non-PD group, $\mathrm{p}<0.001 ; \mathrm{J}-\mathrm{HAQ}$ score, 0.25 in PD group, 0.13 in non-PD group, $p<0.001$ ). Median of delta DAS28 and delta J-HAQ in the both groups were similar and adjusted ORs of PD for DAS28 remission (0.85 [0.69-1.04]) and for J-HAQ remission (0.99 [0.67-1.45]) at 6 month were not statistically significant. There were significant differences in the percentage of patients who developed infections between the two groups $(5.8 \%$ in PD group, 3.4\% in non-PD group, $p=0.002$ ). Adjusted OR of PD for infections was 1.72 [1.10-2.69], which was significantly elevated.

Conclusion: RA patients with PD had similar treatment response with those in the non-PD group, however, had higher disease activity, poorer physical function, and higher risk of infections compared to those without. These results may indicate that oral management is important for the better outcomes of patients with RA in the daily practice.

\section{REFERENCES:}

[1] Nat Rev Rheumatol. 2017:606-20

[2] J Clin Rheumatl. 2012:180-4

[3] Med J Islam Repub. Iran. 2017:44

Acknowledgement: We thank all patients who participated in the IORRA survey and all of the members of the Institute of Rheumatology, Tokyo Women's Medical University, for the successful management of the IORRA cohort. 